$\xi=$

\title{
The Effect of Media Sports Immersion on Smart Phone Users' Loyalty and Sports Activities
}

\author{
Nam-Ik, Kim ${ }^{1}$ and Sun-Mun, Park ${ }^{2} *$ \\ ${ }^{1}$ Department of Physical Education Graduate School, Catholic Kwadong University, Gangneung 25601, Korea. \\ ${ }^{2}$ Department of Sports Leisure Graduate School, Honam University, Gwangju 506-714, Korea. \\ *Corresponding author E-mail:psmun@honam.ac.kr
}

\begin{abstract}
Modern society is an information society through media, which has a great effect on human behavior, and this social flow also has a great influence on people's sports activities. In other words, people can acquire information about sports that they want anywhere and anytime, can express their opinions, and can form groups with people who have same interest due to the development of wireless internet and smart phones. In addition, the immersion in media sports is influential as a major factor of sports activities. The immersion on the internet is defined as an important criterion of consumers' spontaneous interest and as what includes individuals' pleasure, mental stimulation and awareness of interest. The immersion in media sports is connected to loyalty. The loyalty, which is a factor that affects the decision of human behaviors, means favorable attitudinal and mental attachment. It means the loyalty is preceded until sports activities are performed through media sports. Therefore, this study is intended to reveal the effects of media immersion by media sports involving experiences on sports participation. This study is intended to reveal the effects of media immersion by media sports involving experiences on sports participation. For this study, total 300 participants in their 20s $\sim 30$ s including 150 males and 150 females who experienced media sports using smart phones were selected with the convenience sampling method. Prior to the survey, their media sports involving experiences using smart phones were confirmed. For the questionnaire completion method, self-administration method was used. For analysis of data, the data with answers of questionaries completed were collected, valid samples are coded according to the coding manual with double entry and no entry excluded, the coded data were entered into computer individually, and then frequency analysis, Exploratory Factor Analysis, Reliability Analysis and Multiple Regression Analysis were done with the use of SPSS Windows 18.0 Version statistical program. The results of the study are as follows. First, the immersion of media sports has a partial effect on sports loyalty. Second, the immersion of media sports has a partial effect on sports activities. Third, the high sports loyalty has a partial effect on sports activities.
\end{abstract}

Keywords: Media Sports, Sports Activities, sports immersion, loyalty level

\section{Introduction}

Modern society is an information society through media, which has a great effect on human behavior, and this social flow also has a great influence on people's sports activities. That is, people can obtain information about sports that they want anywhere and anytime, can express their opinions, and can form groups with people who have same interest thanks to the development of wireless internet and smart phones.

In addition, the immersion in media sports is influential as a major factor of sports activities. The immersion on the internet is defined as an important criterion of consumers' spontaneous interest and as what includes individuals' pleasure, mental stimulation and awareness of interest[1],[2]. When this social environment is viewed in connection with sports, mass-media and sports are in symbiotic relationship in modern society, so people came to prefer acceptable messages rather than enjoying high level luxury culture in order to escape physical and mental anxiety caused by daily life and to overcome self-alienation. This indicates that the public choose sports and enjoy the culture due to interest, pleasure, thrill, release of emotions, which are the amusement characteristics that sports have[3], [4].
In other words, media is expanding and developing in that people realize their interest through sports and media becomes an important part of communication in sports. In the media industry, sports account for a big part, so a terminology 'media sports' has emerged, which is an interdependent symbiotic relationship and phenomenon of sports and media.

The media sports is a sport medium by media, and it refers to sports phenomena mediated by media. It involves what is related to all the messages including sports knowledge, information and game scenes conveyed to sports fans. It can be also the combination of media and sports.

In modern society, sports is small edition that reflects a society's dominating value and attitude, and the experience of sports activities is known to provide the best means to perform norms and roles owned by a specific society. In actuality, sports has a function of socialization that conveys desirable value to social members. This media sports immersion has an effect on sports activities as a major factor. The immersion on internet is defined as a critical criteria of the degree of consumers' spontaneous interest and the one that includes individuals' pleasure, psychological stimulation, and the awareness of interest[5],[6].

The media sports immersion is connected to loyalty. Loyalty is an important factor that affects the decision of human behaviors, and 
means a favorable attitude and psychological attachment on a specific matter.

The media sports is more actively performed in online environment. This can be found in online communities. People belong to groups where they recognize they have same interest, satisfy their desires interdependently and form community spirit[7],[8].

Sports communities, where people who like sports share information and participate in sports games to promote friendship, have been formed and universalized. That is, loyalty is preceded before sports activities are done through media sports.

As a result, the development of information communication technology and the generalization of internet have made modern people use internet first to obtain useful information to do activities unlike the past.

The field of sports has been changed from the offline oriented activities in the past into online activities. Therefore, this study is intended to find out the effects of media immersion according to experiences of media sports on sports participation.

\section{Research Method}

\subsection{Study Subjects}

Total 300 people( 150 men, 150 women) between 20 and 30 years of age in Seoul and Gwangju who used media sports with smart phones as of 2016 were selected in the convenience sampling method.

Prior to survey, they were checked whether they were involved in media sports using smart phones.

For questionnaire completion method, the self-administration method was used. The general characteristics of the participants are shown in $\langle$ Table 1$\rangle$.

Table 1: General Characteristics of Participants

\begin{tabular}{|c|c|c|c|}
\hline \multicolumn{2}{|c|}{ Variable } & Number of Cases(N) & Percentage(\%) \\
\hline \multirow{2}{*}{ Gender } & male & 160 & 53.3 \\
& female & 140 & 46.7 \\
\hline \multirow{2}{*}{ Age } & $20 \mathrm{~s}$ & 150 & 50 \\
& $30 \mathrm{~s}$ & 150 & 50 \\
\hline \multirow{2}{*}{ Region } & Seoul & 180 & 60 \\
& Gwangju & 120 & 40 \\
\hline
\end{tabular}

\subsection{Survey Tools}

For research tools to measure sports immersion, based on questions used by Novak(1998)[9], five-point scale was constituted with two sub-factors of interest and challenging spirit. To measure loyalty level, based on questions used by Yoo, Jong-Sang and Park, Sun-Mun(2017)[10], five-point scale was constituted with two sub-factors of behavioral loyalty and attitudinal loyalty.

To measure sports activities, based on questions used by Park Soon-moon and Lee A-yeong(2015)[11], [12], five-point scale was constituted with three sub-factors of cognitive, definitional and behavioral participations.

\subsection{Validity and Reliability of Questionnaire}

\subsubsection{Factor Analysis of Media Sports Immersion}

The result of exploratory factor analysis on the validity test of media sports immersion is in $\langle$ Table 2$\rangle$.

Table 2: Factor Analysis on media sports immersion

\begin{tabular}{|c|c|c|c|}
\hline \multicolumn{2}{|c|}{ Question } & Factor 1 & Factor 2 \\
\hline \multirow{3}{*}{ Interest } & Q01 & .804 & .181 \\
& Q03 & .731 & .247 \\
& Q02 & .712 & .230 \\
& Q04 & .615 & .234 \\
\hline
\end{tabular}

\begin{tabular}{|c|c|c|c|}
\hline \multirow{3}{*}{ Sprit of challenge } & Q06 & .089 & .792 \\
& Q07 & .112 & .774 \\
& Q08 & .080 & .723 \\
& Q05 & .133 & .710 \\
& Q09 & .197 & .634 \\
\hline \multicolumn{2}{|c|}{ characteristic value } & 1.757 & 1.636 \\
dispersion \% & 43.915 & 40.889 \\
\multicolumn{2}{|c|}{ accumulation \% } & 43.915 & 84.804 \\
\hline
\end{tabular}

Specifically, the items that show high factor loading amount(over $0.615)$ on Factor 1 are No. 1, 3, 2 and 4, all of which are related to interest.

The items that show high factor loading amount(over 0.634) on Factor 2 are No. 6, 7, 8, 5 and 10, all of which are related to spirit of challenge.

The accumulation rate that explains the two factors of interest and spirit of challenge is approximately $84.804 \%$.

This analysis result indicates that media sports immersion is relatively validly measured.

\subsubsection{Factor Analysis of Loyalty}

The result of exploratory factor analysis of validity test on loyalty is in $\langle$ Table 3$\rangle$.

Table.3: Factor Analysis of Loyalty

\begin{tabular}{|c|c|c|c|}
\hline \multicolumn{2}{|c|}{ Question } & Factor 1 & Factor 2 \\
\hline \multirow{2}{*}{ Behavioral } & Q01 & .841 & .093 \\
& Q02 & .821 & .031 \\
\hline \multirow{2}{*}{ Attitudinal } & Q03 & .022 & .832 \\
& Q04 & .054 & .765 \\
\hline \multicolumn{2}{|c|}{ characteristic value } & 3.344 & 3.028 \\
dispersion \% & 37.151 & 33.640 \\
accumulation \% & 37.151 & 70.791 \\
\hline
\end{tabular}

Specifically, the items that show high factor loading amount(over 0.821 ) on Factor 1 are No. 1 and 2, and both are related to behavioral loyalty. The items that show high factor loading amount(over 0.765 ) on Factor 2 are No. 3 and 4, and both are related to attitudinal loyalty.

The accumulation rate that explains the two factors of behavioral and attitudinal loyalty is approximately $70.791 \%$. This analysis result indicates that loyalty is relatively validly measured.

\subsubsection{Factor Analysis of Sports Activities}

The result of exploratory factor analysis of validity test on sports activities is in $\langle$ Table 4$\rangle$.

Table 4: Factor Analysis of Sports Activities

\begin{tabular}{|c|c|c|c|c|}
\hline \multicolumn{2}{|c|}{ Question } & Factor 1 & Factor 2 & Factor 3 \\
\hline \multirow{4}{*}{ Cognitive } & Q03 & .878 & .021 & .082 \\
& Q02 & .821 & .018 & .125 \\
& Q01 & .768 & .091 & .044 \\
& Q04 & .759 & .117 & .012 \\
\hline \multirow{4}{*}{ Definitional } & Q05 & .180 & .870 & .136 \\
& Q07 & .190 & .714 & .176 \\
& Q08 & .011 & .659 & .100 \\
\hline \multirow{4}{*}{ Behavioral } & Q09 & .101 & .031 & .819 \\
& Q12 & .022 & .149 & .784 \\
& Q10 & .093 & .103 & .702 \\
\hline \multicolumn{2}{|c|}{ characteristic value } & 2.267 & 2.150 & 1.162 \\
dispersion \% & 32.389 & 30.710 & 63.099 \\
accumulation \% & 32.389 & 63.099 & 79.692 \\
\hline \multicolumn{4}{|c|}{}
\end{tabular}

Specifically, the items that show high factor loading amount(over 0.779) on Factor 1 are No. 3, 2, 1 and 4, all of which are related to cognitive activity. The items that show high factor loading amount(over 0.659) on Factor 2 are No. 5, 7 and 8, all of which are related to definitional activity. Question 6 was excluded due to 
low factor loading amount. The items that show high factor loading amount(over 0.702) on Factor 3 are No. 9, 12 and 10, all of which are related to behavioral activity. Question 11 was excluded due to low factor loading amount.

The accumulation rate that explains the three factors of cognitive, definitional and behavioral activities is approximately $79.692 \%$. This analysis result indicates that sports activities are relatively validly measured.

\subsubsection{Analysis of Reliability}

The reliability analysis result is in $\langle$ Table 5>.

Table 5: Reliability Analysis Result of This Study

\begin{tabular}{|c|c|c|}
\hline Variable & Sub-factor & Chronbach's $\alpha$ Value \\
\hline \multirow{2}{*}{ Media sports immersion } & Interest & .79 \\
& Spirit of challenge & .80 \\
\hline \multirow{2}{*}{ Loyalty } & Behavioral & .82 \\
& Attitudinal & .91 \\
\hline \multirow{2}{*}{ Sports activities } & Cognitive & .81 \\
& Definitional & .79 \\
& Behavioral & .80 \\
\hline
\end{tabular}

Specifically, Cronbach's $\alpha$ value of each sub-factor of media sports immersion is .79 .80, and Cronbach's $\alpha$ value of loyalty is .82 .91. Cronbach's $\alpha$ value of sports activities is .79 .81. In this study, Cronbach's $\alpha$ value, which is the reliability coefficient on all the factors, is high, so these are considered as reliable questions.

\subsection{Statistical Analysis}

For data analysis, the questionaries with answers completed were collected, data with double entry or no-entry was excluded, and valid samples were coded according to the guideline of coding. The coded data was input individually into the computer, and then frequency analysis, exploratory factor analysis, reliability analysis and multiple regression analysis were done with the use of SPSS Windows 18.0 Version statistical program.

\section{Results and Discussion}

The purpose of this study is to investigate the effect of media sports commitment on loyalty and sports activities.

The results of the regression analysis are as follows.

\subsection{The Effects of Media Sports Immersion on Loyalty}

The result of multiple regression analysis to find out the effects of media sports immersion on loyalty is in $\langle$ Table 6〉.

Table 6: Multiple regression analysis on the effects of media sports immersion on loyalty

\begin{tabular}{|c|c|c|c|c|}
\hline \multirow{2}{*}{ Variable } & \multicolumn{2}{|c|}{ Behavioral } & \multicolumn{2}{c|}{ Attitudinal } \\
\cline { 2 - 5 } & $\beta$ & $\mathrm{t}$ & $\beta$ & $\mathrm{t}$ \\
\hline Constant & & 15.287 & & 9.716 \\
Interest & .214 & $4.450 * * *$ & .192 & $3.620 * * *$ \\
challenge & .053 & 1.065 & .139 & $2.749 * *$ \\
\hline $\mathrm{R}^{2}$ & \multicolumn{3}{|c|}{.165} & \multicolumn{2}{c|}{.043} \\
\hline $\mathrm{F}$ & \multicolumn{2}{|c|}{$28.910 * * *$} & \multicolumn{2}{c|}{$6.640 * * *$} \\
\hline$* * \mathrm{p}<.01, * * * \mathrm{p}<.001$ & \multicolumn{3}{|c}{} \\
\hline
\end{tabular}

<Table 6> shows that media sports immersion has a statistically significant effect on behavioral and attitudinal loyalty at the level of $0.1 \%$ in the regression equation.

Specifically, just interest has a significant effect on behavioral loyalty at the level of $0.1 \%$, and future health has $5 \%$ of significant effect.
Regarding $\beta$ value, the relative contribution, interest(.214) has more effect than spirit of challenge(.053).

The explanation power of media sports immersion on behavioral loyalty is $16.57 \%$ of total variable.

Interest has a significant effect on attitudinal loyalty of media sports immersion at the level of $0.1 \%$, and spirit of challenge has $1 \%$ of significant effect. Regarding $\beta$ value, the relative contribution, interest(.192) has more effect than spirit of challenge(.139).

The explanation power of media sports immersion on attitudinal loyalty is $4.3 \%$ of total variable.

\subsection{The Effects of Media Sports Immersion on Sports Activities}

The result of multiple regression analysis to find out the effects of media sports immersion on sports activities is in $\langle$ Table 7$\rangle$.

Table 7: Multiple regression analysis on the effects of media sports immersion on sports activities

\begin{tabular}{|c|c|c|c|c|c|c|}
\hline \multirow{2}{*}{ Variable } & \multicolumn{2}{|c|}{ Cognitive } & \multicolumn{2}{|c|}{ Definitional } & \multicolumn{2}{|c|}{ Behavioral } \\
\hline & $\beta$ & $\mathrm{t}$ & $\beta$ & $\mathrm{t}$ & $\beta$ & $\mathrm{t}$ \\
\hline $\begin{array}{l}\text { Constant } \\
\text { Interest } \\
\text { challenge }\end{array}$ & $\begin{array}{l}.391 \\
.328\end{array}$ & $\begin{array}{c}4.335 \\
9.356^{* * *} \\
7.987^{* * *}\end{array}$ & $\begin{array}{l}.385 \\
.009\end{array}$ & $\begin{array}{c}6.145 \\
9.180^{* * *} \\
.202\end{array}$ & $\begin{array}{l}.282 \\
.422\end{array}$ & $\begin{array}{c}7.523 \\
6.669 * * * \\
10.180^{* * * *}\end{array}$ \\
\hline $\mathrm{R}^{2}$ & \multicolumn{2}{|r|}{.124} & \multicolumn{2}{|r|}{.365} & \multicolumn{2}{|r|}{.356} \\
\hline $\mathrm{F}$ & \multicolumn{2}{|c|}{$85.392 * * *$} & \multicolumn{2}{|c|}{$83.948 * * *$} & \multicolumn{2}{|c|}{$35.070 * * *$} \\
\hline
\end{tabular}

$<$ Table 7> shows that media sports immersion has a significant effect on cognitive, definitional and behavioral activities at the level of $0.1 \%$ in the regression equation.

Specifically, interest and spirit of challenge have a significant effect on cognitive activities at the level of $0.1 \%$. Regarding $\beta$ value, the relative contribution, interest(.391) has a more effect than spirit of challenge(.328).

The explanation power of media sports immersion on cognitive activities is $12.4 \%$ of total variable.

Just interest has a significant effect on definitional activities at the level of $0.1 \%$. Regarding $\beta$ value, the relative contribution, interest(.385) has a more effect than spirit of challenge(.009).

The explanation power of media sports immersion on definitional activities is $36.5 \%$ of total variable.

Interest and spirit of challenge have a significant effect on behavioral activities at the level of $0.1 \%$. Regarding $\beta$ value, the relative contribution, spirit of challenge(.422) has a more effect than interest(.282).

The explanation power of media sports immersion on behavioral activities is $36.5 \%$ of total variable.

Just interest has a significant effect on definitional activities at the level of $0.1 \%$. Regarding $\beta$ value, the relative contribution, interest(.385) has a more effect than spirit of challenge(.009).

The explanation power of media sports immersion on definitional activities is $36.5 \%$ of total variable.

\subsection{The Effects of Loyalty on Sports Activities}

The result of multiple regression analysis to find out the effects of loyalty on sports activities is in $\langle$ Table 8$\rangle$.

Table 8: Multiple regression analysis on the effects of loyalty on sports activities

\begin{tabular}{|c|c|l|c|l|c|l|}
\hline \multirow{2}{*}{ Variable } & \multicolumn{2}{|c|}{ Cognitive } & \multicolumn{2}{c|}{ Definitional } & \multicolumn{2}{c|}{ Behavioral } \\
\cline { 2 - 7 } & $\beta$ & \multicolumn{1}{c|}{$\mathrm{t}$} & $\beta$ & \multicolumn{1}{c|}{$\mathrm{t}$} & $\beta$ & \multicolumn{1}{c|}{$\mathrm{t}$} \\
\hline Constant & & 38.603 & & 63.123 & & 61.504 \\
Behavioral & .399 & $8.922 * * *$ & .561 & $13.640 * * *$ & .558 & $13.480 * * *$ \\
Attitudinal & .223 & $5.003 * * *$ & .145 & $3.522 * * *$ & .184 & $4.448 * * *$ \\
\hline $\mathrm{R}^{2}$ & \multicolumn{2}{|c|}{.120} & \multicolumn{2}{|c|}{.255} & \multicolumn{2}{c|}{.243} \\
\hline $\mathrm{F}$ & \multicolumn{2}{|c|}{$40.004 * * *$} & \multicolumn{2}{c|}{$100.198 * * *$} & \multicolumn{2}{c|}{$94.298 * * *$} \\
\hline
\end{tabular}


$<$ Table 8> shows that loyalty has a statistically significant effect on cognitive, definitional and behavioral activities at the level of $0.1 \%$ in the regression equation.

Specifically, behavioral loyalty and attitudinal loyalty have a significant effect on cognitive activities at the level of $0.1 \%$. Regarding $\beta$ value, the relative contribution, behavioral loyalty(.399) has a more effect than attitudinal loyalty(.223).

The explanation power of loyalty on cognitive activities is $12 \%$ of total variable.

Behavioral loyalty and attitudinal loyalty have a significant effect on definitional activities at the level of $0.1 \%$. Regarding $\beta$ value, the relative contribution, behavioral loyalty(.561) has a more effect than attitudinal loyalty(.145).

The explanation power of loyalty on definitional activities is $25.5 \%$ of total variable.

Behavioral loyalty and attitudinal loyalty have a significant effect on behavioral activities at the level of $0.1 \%$. Regarding $\beta$ value, the relative contribution, behavioral loyalty $(.558)$ has a more effect than attitudinal loyalty(.184).

The explanation power of loyalty on behavioral activities is $24.3 \%$ of total variable.

\section{Conclusion}

This study is intended to find out the relation of media sports immersion with loyalty and sports activities targeting those in their 20s and 30s who have been involved in media sports in Seoul and Gwangju.

The results of the study are as follows.

First, media sports immersion has a partial effect on loyalty.

In other words, as the interest level of media sports immersion is highly recognized, behavioral loyalty is high. As interest and spirit of challenge are highly recognized, attitudinal loyalty is high.

Second, media sports immersion has a partial effect on sports activities.

As the interest and spirit of challenge are highly recognized, the levels of cognitive and behavioral activities are high. As spirit of challenge is highly recognized, the level of definitional activities is high.

Third, high sports loyalty has a partial effect on sports activities.

That is, as behavioral loyalty and attitudinal loyalty are high, cognitive, definitional and behavioral activities are high.

\section{References}

[1] J. D. Kim, and T. H., Jeon, An Effective Solution for the Multimedia Telephony Services in Evolving Networks, International Journal of Advanced Smart Convergence(IJASC), 2016, 2(1), 24-26.

[2] M. R. Kwon, The Impact of Emotional Intelligence and SelfEsteem on Internet and Smart Phone Addiction, Sports Activities, and Reading of Youth. International Journal of Internet, Broadcasting and Communication (JIBC), (2017), 9(1), 35-41.

[3] S. Y. Hong, and H. O. Kim, Ann M. S, Lee S, Y, Cha J. S., A Study on description method of product information by utilizing a display specific for store support. International Journal of Advanced Smart Convergence (IJASC), 2015, 4(2), 79-83.

[4] S. M. Park, and A. H. Lee, An Influence of the Using Pattern of Sports Media on Loyalty \& Sports Attitude, Journal of International Information Institute, (2016), 19(9a), 1344-8994.

[5] S. Y. Kim, The Influence of the Characteristic of Information Providers on the Intention to Accept Information, Soongsili University, (2013), 84-94.

[6] J. H. Kim, and J. H. Lee, The Effect of Three Broadcasting Station Sports Specialized Channels Commentator's Credibility on Channel Satisfaction and Channel Loyalty, (2011), 16(5), pp. 17-28.

[7] S. H. Han, Characteristics and Comparison of Popular Channels on Internet Game Broadcasting: Focus on Twitch TV. The Journal of The Institute of Internet, Broadcasting and Communication (JIBC), (2016), 16(4), 7-14.

[8] J. H. Hwang, "The Relationship among Contacting Motivation, Involvement Experience Sport Attitude and Sport Participation of
Media Sports", Korean Journal of Sport Science. 23(6), pp. 664676, 2013.

[9] T. P. Novak, Modeling the Structure of the Flow Experience Among Web Users of Sport as Perceived by the General Population. International Review ofsportSociology, 10, (1998).

[10] J. S. Yoo and S. M. Park, The Effect of Internet Media Ability on Interactivity and Sports Site Loyalty, Journal of Engineering and Applied Sciences, (2017), 12(10), 2556-2559.

[11] N. I. Kim, and S. M. Park, The Relationship between Media Sports Involvement Experiences and Sports Values and Sports Participation, International Journal of Applied Engineering Research, (2017), 12(20), 9768-9773.

[12] A. H. Lee, and S. M. Park, Relation between Online Sports Community Awareness and Sports Activity, Journal of The Institute of Internet, Broadcasting and Communication (IIBC), (2015), 18(10), $1343-4500$. 